Received 00th January 20xx, Accepted 00th January 20xx DOI: $10.1039 / x 0 \times x 00000 x$

\title{
Sustainable strategies based on glycine-betaine analogues ionic liquids for the recovery of monoclonal antibodies from cell culture supernatants
}

\author{
Emanuel V. Capela, ${ }^{a}{ }^{a b}$ Alexandre E. Santiago, ${ }^{a}$ Ana F.C.S. Rufino, ${ }^{a}$ Ana P.M. Tavares, ${ }^{a}$ Matheus M. \\ Pereira, ${ }^{a}$ Aminou Mohamadou, ${ }^{c}$ M. Raquel Aires-Barros, ${ }^{b}$ João A.P. Coutinho, ${ }^{a}$ Ana M. Azevedo, ${ }^{b}$ \\ and Mara G. Freire*a
}

\begin{abstract}
Monoclonal antibodies (mAbs) are of crucial interest for therapeutic purposes, especially in vaccination, immunization, and in the treatment of life-threatening diseases. However, their downstream processing from the complex cell culture media in which they are produced still requires multiple steps, rendering mAbs as extremely high-cost products. Therefore, the development of cost-effective, sustainable and biocompatible purification strategies for mAbs is in high demand to decrease the associated economic, environmental and health burdens. Herein, novel aqueous biphasic systems (ABS) composed of glycine-betaine analogues ionic liquids (AGB-ILs) and $\mathrm{K}_{2} \mathrm{HPO}_{4} / \mathrm{KH}_{2} \mathrm{PO}_{4}$ at $\mathrm{pH} 7.0$, the respective three-phase partitioning (TPP) systems, and hybrid processes combining ultrafiltration, were investigated and compared in terms of performance as alternative strategies for the purification and recovery of anti-interleukin-8 (anti-IL-8) mAbs, which are specific therapeutics in the treatment of inflammatory diseases, from Chinese Hamster Ovary $(\mathrm{CHO})$ cell culture supernatants. With the studied ABS, mAbs preferentially partition to the IL-rich phase, with recovery yields up to $100 \%$ and purification factors up to 1.6 . The best systems were optimized in what concerns the IL concentration, allowing to take advantage of IL-based three-phase partitioning approaches where a precipitate enriched in mAbs is obtained at the ABS interface, yielding $41.0 \%$ of IgG with a purification factor of 2.7 (purity of $60.9 \%$ ). Hybrid processes combining the two previous techniques and an ultrafiltration step were finally applied, allowing the recovery of mAbs from the different fractions in an appropriate buffer solution for further biopharmaceuticals formulation, while allowing the simultaneous IL removal and reuse. The best results were obtained with the hybrid process combining TPP and ultrafiltration, allowing to obtain mAbs with a purity of $67.2 \%$. The biological activity of anti-IL-8 mAbs is maintained after the several purification and recovery steps, indicating that the novel ABS, three-phase partitioning and hybrid processes comprising AGB-ILs are promising and sustainable strategies in mAbs downstream processing.
\end{abstract}

\section{Introduction}

Despite all significant advances that have been accomplished in the development of effective therapies, biopharmaceuticals are in many cases the unique option in the treatment of particular diseases. $^{1-2}$ Amongst biopharmaceuticals, monoclonal

\footnotetext{
a. CICECO - Aveiro Institute of Materials, Department of Chemistry, University of Aveiro, 3810-193 Aveiro, Portugal.

b. iBB - Institute for Bioengineering and Biosciences, Department of Bioengineering, Instituto Superior Técnico, Universidade de Lisboa, Av. Rovisco Pais, 1049-001 Lisbon, Portugal.

c. ICMR - Institute of Molecular Chemistry of Reims, University of Reims Champagne Ardenne, CNRS UMR 7312, UFR des Sciences Exactes et Naturelles, BP 1039, F51687 Reims cedex 2, France.

* Corresponding author - E-mail: maragfreire@ua.pt; Fax: +351 234370084; Tel: $+351234370200$

+ Electronic Supplementary Information (ESI) available: detailed information of the upstream process, phase diagrams, TLs and TLLs determination and CELISA assay; experimental binodal weight fraction, TLs and TLLs data; correlation parameters to describe the experimental binodal curve; performance parameters of $A B S$ and $A B S$ + UF processes towards mAbs; water content of the ABS' phases; macroscopic aspect of the extraction ABS; SE-HPLC chromatographic profiles. See DOI: $10.1039 / x 0 x x 00000 x$
}

antibodies (mAbs) are widely applied for therapeutic purposes, namely in vaccination and immunization, but also in the treatment of oncologic, autoimmune, cardiovascular, inflammatory and neurological diseases.1,3 The first therapeutic monoclonal antibody approved, in 1986 by the US Food and Drug Administration (FDA), was Muromonab (Orthoclone OKT3), which is an in vivo produced mAb by hybridoma cells for the prevention of kidney transplant rejection. ${ }^{4}$ By the end of 2017, 57 therapeutic mAbs were approved by the FDA and the European Medicines Agency (EMA) for therapeutic purposes. ${ }^{5}$ In 2017, the worldwide mAbs market exceeded US\$98 billion in sales, representing the majority of total sales in the pharmaceutical market, while aiming to reach US\$ 137-200 billion in 2022.4, 5 Despite all advantages of mAbs, the high quantities required of pure mAbs and their extremely high manufacturing costs, mainly derived from the multiple downstream processing steps, are the most challenging features limiting their widespread use. ${ }^{6}$ The bioprocessing of mAbs comprises 2 steps: (i) upstream processing, based on biological processes where mAbs production occurs through cell cultures derived from mammalian cells; and (ii) downstream 
processing, where the recovery, purification and isolation of mAbs from the complex medium takes place. The upstream processing suffered significant improvements in recent years and the main current bottleneck in mAbs production conveys in the downstream processing. ${ }^{7}$ The current downstream platform includes several steps, including: (i) clarification of the supernatant, (ii) capture of mAbs, (iii) viral inactivation, (iv) polishing of mAbs, (v) viral removal and (vi) concentration/formulation of the final mAb-product. ${ }^{1,8}$ Protein A (ProA) affinity chromatography is the "gold standard" approach in the capture and purification steps since it is highly selective. ${ }^{9}$ However, it presents several limitations, namely the low $\mathrm{pH}$ values necessary to elute $\mathrm{mAb}$-products that can cause their aggregation, the presence of some impurities in the final formulation since the ProA ligand can be degraded by proteases present in the supernatants, and the high cost of the resin (between US\$5,000 and US\$15,000/L; industrial columns require volumes up to $1000 \mathrm{~L}) .9,10$ Accordingly, ProA affinity chromatography is the main centre of costs of the mAbs manufacturing process, with the overall downstream processing contributing up to $80 \%$ of the mAbs global production costs. ${ }^{1,11}$ Therefore, it is imperative to develop sustainable and cost-effective downstream processes for mAbs to decrease their cost and to allow their widespread use.

Aqueous biphasic systems (ABS) were first proposed as an extraction technique by Albertsson ${ }^{12}$, being typically composed of two polymers, a polymer and a salt or two salts dissolved in aqueous media (ternary systems), which above given concentrations cause phases to split. Their physicochemical characteristics can be tailored by changing the phase-forming components and their concentration, $\mathrm{pH}$ and temperature to profit the selective partitioning of a target compound.1,13 One of the major advantages of $A B S$ over other techniques commonly applied to the recovery of mAbs is that the clarification, extraction, purification, and concentration steps can be integrated in a single step. Furthermore, ABS are rich in water, do not require the use of volatile organic solvents, and represent a technique easy to scale up and able to operate in continuous mode. ${ }^{13,} 14$ Thus, if properly designed, ABS are effective, sustainable and low-cost strategies when compared to the applied chromatographic methods. Traditional ABS formed by polymers have been studied for the purification of IgG (including mAbs). ${ }^{1}$ However, the restricted polarity presented by this type of systems compromises good recovery yields and purity factors to be achieved, thus encouraging the development of several strategies to overcome this bottleneck. The addition of salts, ${ }^{15}$ affinity ligands ${ }^{13}, 14$ and the use of modified phase-forming components ${ }^{16}$ are some examples in this field. In addition to more traditional polymer-based $A B S$, ionic-liquid-(IL)-based ABS emerged in more recent years with the pioneering work of Rogers and co-workers, ${ }^{17}$ and since then have been investigated as extraction/purification platforms for a plethora of (bio)molecules. ${ }^{18-23}$ By using ILs as phase-forming components of ABS it is possible to tailor the phases' polarities and affinities to specific bioproducts, overcoming the limited polarity presented by traditional polymer-based systems. ${ }^{18,24}$ Given the designer solvents ability of ILs, they can thus be foreseen as promising phase-forming components to carry out the purification and recovery of mAbs. To the best of our knowledge, there are currently no reports in the literature considering the mAbs recovery from $\mathrm{CHO}$ cell culture supernatants using ILs as phase-forming components of ABS.

Within the field of IL-based ABS, ionic-liquid-based three-phase partitioning (ILTPP) have been investigated for the recovery of proteins, ${ }^{25}, 26$ yet never considered for the purification of antibodies. Typically, three-phase partitioning (TPP) approaches involve the recovery of the target protein in an enriched precipitate at the interface of two liquid phases. ${ }^{27}$ Alvarez et al. ${ }^{25,} 26$ demonstrated the feasibility of this approach using ILbased ABS, proposing the concept of ionic-liquid-based TPP (ILTPP), but still only applied to food proteins and less complex matrices.

Even though ILs present interesting environmental features compared to volatile organic compounds, mainly due to their negligible vapor pressure at ambient conditions, their toxicity and biodegradability should be always considered. ${ }^{28,} 29$ In order to overcome these drawbacks, glycine-betaine analogues ILS (AGB-ILs) were here synthesized and investigated as phaseforming components of ABS and TPP approaches. Glycinebetaine and its analogous are usual natural components of the mammalian diet, being present in fruits, vegetables, and coffee beans. ${ }^{30}$ In particular, novel ABS and TPP systems formed by AGB-ILs and $\mathrm{K}_{2} \mathrm{HPO}_{4} / \mathrm{K}_{2} \mathrm{HPO}_{4}$ at $\mathrm{pH} 7.0$ were investigated to purify and recover biopharmaceuticals, using anti-human interleukin-8 (anti-IL-8) mAbs from Chinese Hamster Ovary (CHO) cell culture supernatants. These antibodies are potential therapeutics to treat inflammatory diseases. ${ }^{31-33}$ Given their novelty, the ABS phase diagrams were determined to ascertain the compositions required to form two-phase and TPP systems able to act as separation techniques. Then, their potential in the extraction and purification of mAbs was evaluated and optimized, either as one-step platforms, as three-phase partitioning systems, or as hybrid processes combined with ultrafiltration, followed by the evaluation of the mAbs specific activity.

\section{Materials and methods}

Material, chemicals and biologicals. AGB-ILs were synthesized by us according to previously reported protocols, ${ }^{34}$ corresponding to the following ILs: triethyl[4-ethoxy-4-oxobutyl]ammonium bromide $\quad\left(\left[\mathrm{Et}_{3} \mathrm{NC}_{4}\right] \mathrm{Br}\right), \quad$ tri( $n$-propyl)[4-ethoxy-4oxobutyl]ammonium bromide $\left(\left[\mathrm{Pr}_{3} \mathrm{NC}_{4}\right] \mathrm{Br}\right), \quad$ tri $(n$-butyl $)[4-$ ethoxy-4-oxobutyl]ammonium bromide $\left(\left[\mathrm{Bu}_{3} \mathrm{NC}_{4}\right] \mathrm{Br}\right)$ and $\mathrm{N}$-(1methylpyrrolidyl-4-ethoxy-4-oxobutyl)ammonium bromide ([MepyrNC $\left.\mathrm{M}_{4}\right] \mathrm{Br}$ ). ILs were synthesized by the reaction of 4bromobutyrate acid ethyl ester and triethylamine, tri( $n$ propyl)amine, tri(n-butyl)amine or 1-methylpyrrolidine, respectively. All ILs were dried under vacuum for at least $72 \mathrm{~h}$ at $45^{\circ} \mathrm{C}$. After this procedure, the purity of each IL was checked by ${ }^{1} \mathrm{H}$ and ${ }^{13} \mathrm{C}$ nuclear magnetic resonance (NMR), being $>97 \%$. All ILs synthesized are solid at room temperature, yet with melting points below $100^{\circ} \mathrm{C}$, and are water soluble at $25^{\circ} \mathrm{C} \cdot{ }^{34}$ The 
commercial ILs studied in this work for comparison purposes are the following: tetra( $n$-butyl)ammonium bromide $\left(\left[\mathrm{N}_{4444}\right] \mathrm{Br}, 98\right.$ $\%$ purity) and 1-butyl-3-methylimidazolium bromide ([ $\left.\mathrm{C}_{4} \mathrm{mim}\right] \mathrm{Br}, 99 \%$ purity), acquired from Fluka and Iolitec, respectively. The chemical structures of the synthesized and commercial ILs are depicted in Figure 1.

AGB-ILS

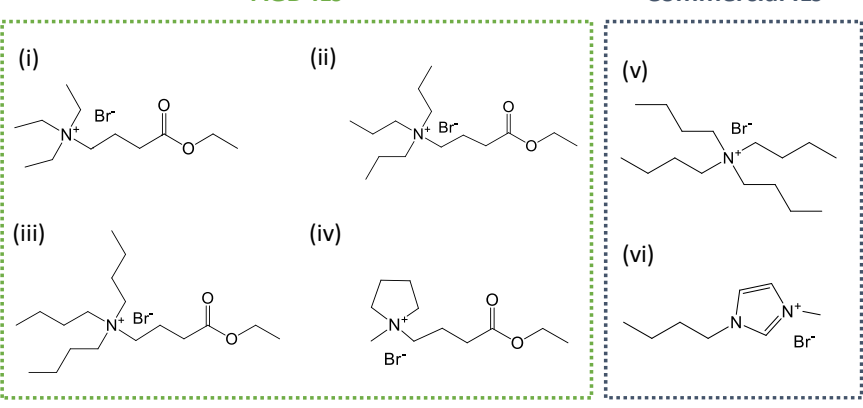

Figure 1. Chemical structures of the investigated ILs: (i) $\left[\mathrm{Et}_{3} \mathrm{NC}_{4}\right] \mathrm{Br}$; (ii) $\left[\mathrm{Pr}_{3} \mathrm{NC}_{4}\right] \mathrm{Br}$; (iii) $\left[\mathrm{Bu}_{3} \mathrm{NC}_{4}\right] \mathrm{Br}$; (iv) [MepyrNC $\left.\mathrm{M}_{4}\right] \mathrm{Br}$; (v) $\left[\mathrm{N}_{4444}\right] \mathrm{Br}$; (vi) $\left[\mathrm{C}_{4} \mathrm{mim}\right] \mathrm{Br}$.

A mixture of the salts $\mathrm{K}_{2} \mathrm{HPO}_{4}$ and $\mathrm{KH}_{2} \mathrm{PO}_{4}$ at $\mathrm{pH} 7.0$ was used for the formation of $A B S$ and TPP systems with AGB-ILs. $\mathrm{K}_{2} \mathrm{HPO}_{4} \cdot 3 \mathrm{H}_{2} \mathrm{O}$ extra pure was acquired from Scharlau, and $\mathrm{KH}_{2} \mathrm{PO}_{4}$ extra pure was acquired from Roic Farma, S.A. Phosphate buffered saline solution (PBS, $\mathrm{pH}=7.4$ ) pellets were acquired from Sigma-Aldrich.

Human IL-8 (77aa) (CXCL8) standard (98\% purity) was acquired from Sigma-Aldrich. Commercial human immunoglobulin $G$ (IgG) for therapeutic administration (trade name: Gammanorm ${ }^{\circledR}$ ) was obtained from Octapharma (Lachen, Switzerland), as a $165 \mathrm{~g} \cdot \mathrm{L}^{-1}$ solution containing $95 \%$ of IgG (of which $59 \%$ correspond to $\operatorname{lgG} 1,36 \%$ to $\lg$ G2, $4.9 \%$ to $\operatorname{lgG} 3$ and $0.5 \%$ to $\operatorname{lgG} 4$ ). Bovine serum albumin (BSA) standards ( $2 \mathrm{~g} \cdot \mathrm{L}^{-1}$ ) were purchased from Thermo Scientific Pierce.

Anti-human interleukin-8 (anti-IL-8) monoclonal antibodies were produced in-house by a CHO DP-12 clone\#1934 (ATCC CRL-12445) from the American Type Culture Collection (LGC Standards, Middlesex, UK), and grown in a mixture of $75 \%(\mathrm{v} / \mathrm{v})$ of serum-free media and $25 \%(\mathrm{v} / \mathrm{v})$ of Dulbecco's modified Eagle's medium (DMEM). This culture was maintained for several months, with the mAbs concentration varying between 40.5 and $99.4 \mathrm{mg} \cdot \mathrm{L}^{-1}$. The produced anti-IL-8 $\mathrm{mAb}$ has an isoelectric point ( $\mathrm{pl}$ ) of 9.3.35 Additional information on the mAbs upstream processing is provided in the ESIt.

Determination of ABS phase diagrams, TLs and TLLs. The ternary phase diagrams of the investigated $A B S$ were initially determined to identify the mixture compositions where two phases can be formed, so that the extraction conditions could be defined for the recovery of mAbs. The determination of the binodal curves was performed using the cloud point titration method at $25( \pm 1){ }^{\circ} \mathrm{C}$ and atmospheric pressure, where the mixture compositions were gravimetrically determined. ${ }^{36}$ The experimental binodal curves were adjusted by the equation proposed by Merchuk et al. ${ }^{37}$ (given in the ESIt). The tie-lines (TLs), which give the coexisting phases compositions for a given mixture point, and respective lengths (tie-line lengths, TLLs), were determined according to lever-arm rule originally proposed by Merchuk et al. ${ }^{37}$ For each ternary phase diagram, 3 TLs were determined, including the mixture compositions at which the extractions of IgG were carried out. Further details for the determination of TLs and TLLs are given in the ESIt. In all studied systems, the top phase is majorly enriched in the IL, whereas the bottom phase is mainly composed of the salt and water.

Recovery of anti-IL-8 mAbs from CHO cell culture supernatants. For the recovery of anti-IL-8 mAbs directly from $\mathrm{CHO}$ cell culture supernatants, ABS and TPP systems with $2.0 \mathrm{~g}$ of total weight were prepared with a fixed concentration of phosphate salt (15 wt\%), combined with (i) 25 wt\%, (ii) 30 wt\% and (iii) $40 \mathrm{wt} \%$ of IL. CHO cell culture supernatant was loaded at $37.5 \mathrm{wt} \%$ in all systems, where the remaining amount to complete each composition corresponds to distilled water. Each ABS and TPP was prepared at least in duplicate. Each mixture was stirred, centrifuged for $3 \mathrm{~min}$ at $112 \times \mathrm{g}$, and left to equilibrate for 30 $\min$ at $25^{\circ} \mathrm{C}$ in order to achieve the complete partitioning or separation of mAbs from the remaining proteins. The volume and weight of the phases and the macroscopic aspect of each extraction system were registered, and both phases were carefully separated. In the cases in which a precipitate was observed in the ABS interface, i.e. for the TPP systems, the precipitate was completely isolated from the remaining phases and resuspended in PBS aqueous solution for further analysis. The $\mathrm{pH}$ values of each phase at $25( \pm 1){ }^{\circ} \mathrm{C}$ were determined using a Mettler Toledo U402-M3-S7/200 micro electrode, showing that a pH $7.0 \pm 0.2$ was maintained in all systems.

The best identified systems to purify mAbs from the cell culture supernatant were subjected to an additional ultrafiltration step aiming at recovering $\mathrm{mAbs}$ in PBS aqueous solution, while allowing the IL removal and reuse. The IL-rich phase and the precipitate of the ABS composed of $40 \mathrm{wt} \%\left[\mathrm{Bu}_{3} \mathrm{NC}_{4}\right] \mathrm{Br}+15 \mathrm{wt} \%$ $\mathrm{K}_{2} \mathrm{HPO}_{4} / \mathrm{KH}_{2} \mathrm{PO}_{4}(\mathrm{pH} 7)+37.5 \mathrm{wt} \% \mathrm{CHO}$ cell culture supernatant $+7.5 \mathrm{wt} \% \mathrm{H}_{2} \mathrm{O}$ were placed in a microcentrifuge tube with an Amicon Ultra-0.5 device containing a cut-off filter of $100 \mathrm{kDa}$ (aiming the simultaneous IL recovery, buffer exchange, and to improve the purification factor by separating lower molecular weight proteins), and centrifuged at $14000 \times \mathrm{g}$ for $15 \mathrm{~min}$. The filtrated solution was collected and phosphate buffer aqueous solution was added. This procedure was repeated for two times to assure the maximum removal of the IL. To recover the retentate concentrated solution, the Amicon ${ }^{\circ}$ Ultra-0.5 filter device was placed upside down in a clean microcentrifuge tube and centrifuged at $1000 \times \mathrm{g}$ for $2 \mathrm{~min}$, and then PBS aqueous solution was added. Both the retentate and the filtrate were analyzed. 
IgG and protein impurities were quantified in all feeds and in each $A B S$ phase by size-exclusion high-performance liquid chromatography (SE-HPLC). Samples were diluted at a 1:2 (v:v) ratio in an aqueous potassium phosphate buffer solution (100 $\mathrm{mmol} \cdot \mathrm{L}^{-1}, \mathrm{pH} 7.0$, with $\mathrm{NaCl} 0.3 \mathrm{~mol} \cdot \mathrm{L}^{-1}$ ) used as the mobile phase. The equipment used was a Chromaster HPLC system (VWR Hitachi) equipped with a binary pump, column oven (operating at $40{ }^{\circ} \mathrm{C}$ ), temperature controlled auto-sampler (operating at $10^{\circ} \mathrm{C}$ ), DAD detector and a column Shodex Protein $\mathrm{KW}-802.5(8 \mathrm{~mm} \times 300 \mathrm{~mm})$. The mobile phase was run isocratically with a flow rate of $0.5 \mathrm{~mL} \cdot \mathrm{min}^{-1}$ and the injection volume was $25 \mu \mathrm{L}$. The wavelength was set at $280 \mathrm{~nm}$. The calibration curve was established with commercial human IgG, from 5 to $200 \mathrm{mg} \cdot \mathrm{L}^{-1}$. Blank systems without biological sample were also prepared to address the interferences of the ABS phase-forming compounds.

The ABS performance was evaluated by the recovery yield and purification factor or purity level for IgG. The recovery yield (\%Yield ${ }_{\mathrm{IgG}}$ ) in the top (IL-rich) phase was determined according to the following equation:

$\%$ Yield $_{I g G}=\frac{[I g G]_{T O P} \times V_{\text {TOP }}}{[I g G]_{\text {initial }} \times V_{\text {initial }}} \times 100$

where $[I g G]_{T O P}$ and $[I g G]_{\text {initial }}$ represent the IgG concentration in the top phase and the initial concentration of IgG in the $\mathrm{CHO}$ cell culture supernatant, respectively, and $V_{T O P}$ and $V_{\text {initial }}$ correspond to the volumes of the top phase and cell culture supernatant loaded in the system, respectively.

In the cases where a precipitate of proteins at the ABS interface occurs, corresponding to the ILTPP approach, the \%Yield ${ }_{I g G}$ in the precipitate was determined according to the following equation:

$$
\% Y_{\text {Yield }}{ }_{I g G}=\frac{[I g G]_{P P} \times V_{\text {final }}}{[I g G]_{\text {initial }} \times V_{\text {initial }}} \times 100
$$

where $[I g G]_{P P}$ and $V_{\text {final }}$ represent the IgG concentration and the final volume of the solution after resuspension, respectively. The percentage purity level of IgG (\% Purity ${ }_{\lg G}$ ) was calculated by dividing the HPLC peak area of IgG $\left(A_{l g G}\right)$ by the total area of the peaks corresponding to all proteins present in the respective sample $\left(A_{\text {Total }}\right)$ :

$$
\% \text { Purity }{ }_{I g G}=\frac{A_{I g G}}{A_{\text {Total }}} \times 100
$$

The purification factor of IgG $\left(P F_{l g G}\right)$ was calculated according to Equation 4:

$\boldsymbol{P F}_{\text {IgG }}=\frac{\% \text { Purity }_{\text {IgG }} \text { phase } / \text { precipitate }}{\% \text { Purity }_{\text {IgG } \text { initial }}}$

where \%Purity IgG phase/precipitate and \%Purity IgG inital $_{\text {correspond to }}$ the purity level of IgG in the top phase of each system or in the precipitate (when the ILTPP studies are being carried out) and the purity level of IgG in the cell culture supernatant, respectively.

The same type of analysis, comprising the determination of the recovery yield, purity level and purification factor, was performed after the ultrafiltration step (hybrid process). The overall yield of the two-step process was calculated according to Equation 5:

$\%$ Yield $_{\text {overall }}=\frac{\% \text { Yield }_{A B S / T P P} \times \%_{\text {Yield }}}{100}$

where $\% Y_{\text {Yield }}$ ABS/TPP and \%Yield ${ }_{U F}$ represent the recovery yield of IgG in the ABS or in the TPP approach and ultrafiltration step, respectively.

Proteins profile and anti-IL-8 mAbs integrity and activity. Sodium dodecyl sulphate polyacrylamide gel electrophoresis (SDSPAGE) assays were performed to infer the proteins profile of each fraction, and to address the anti-IL-8 mAbs integrity/stability after downstream processing. Samples were prepared and diluted in a sample buffer from Bio-Rad containing $62.5 \mathrm{mM}$ Tris $-\mathrm{HCl}, \mathrm{pH} \quad 6.2,2 \%$ SDS, $0.01 \%$ bromophenol blue and $10 \%$ glycerol, under reducing conditions with $100 \mathrm{mM}$ dithiothreitol (DTT) and then denaturated at $100^{\circ} \mathrm{C}$ for $10 \mathrm{~min}$. Gels were further stained with Coomassie Brilliant Blue. Further details are given in the ESIt.

Competitive enzyme linked immunosorbent assays (ELISA) were performed for the $\mathrm{CHO}$ cell culture supernatant and for the top (IL-rich) phase and retentate after the ultrafiltration step in order to evaluate the activity of anti-human IL-8 mAbs after the studied recovery processes. The assay was conducted using a 96-well ELISA plate from a Quantikine ${ }^{\circledR}$ Human IL-8/CXCL8 kit from R\&D systems (Minnneapolis, MN, USA). Further details are given in the ESIt

\section{Results and discussion}

\section{Characterization of ABS and TPP systems}

Novel ABS and TPP systems composed of ILs $+\mathrm{KH}_{2} \mathrm{PO}_{4} / \mathrm{K}_{2} \mathrm{HPO}_{4}$ $\left(\mathrm{pH}\right.$ 7.0) $+\mathrm{H}_{2} \mathrm{O}$ were studied as alternative purification and recovery routes for proteins using anti-IL- $8 \mathrm{mAbs}$ from $\mathrm{CHO}$ cell culture supernatants. AGB-ILs were chosen due to their biocompatible features, whereas the potassium phosphate buffer salt was used to maintain the $\mathrm{pH}$ of the overall $A B S$ at physiological conditions, providing thus an appropriate environment for proteins and simultaneously being able to be used in IgG formulations envisaging their therapeutic applications.

The ABS phase diagrams, and respective tie-lines and length, were initially determined at $25^{\circ} \mathrm{C}$ and atmospheric pressure to characterize the systems and assess the minimum amounts required of each phase-forming component to be used in the investigated separation processes. The AGB-ILs studied correspond to $\left[\mathrm{Et}_{3} \mathrm{NC}_{4}\right] \mathrm{Br}, \quad\left[\mathrm{Pr}_{3} \mathrm{NC}_{4}\right] \mathrm{Br}, \quad\left[\mathrm{Bu}_{3} \mathrm{NC}_{4}\right] \mathrm{Br}$ and [MepyrNC $\left.\mathrm{M}_{4}\right] \mathrm{Br}$, whereas the commercially available ILs studied 
for comparison purposes are $\left[\mathrm{C}_{4} \mathrm{mim}\right] \mathrm{Br}$ and $\left[\mathrm{N}_{4444}\right] \mathrm{Br}-c f$. Figure 1 with the ILs chemical structures. Figure 2 depicts the binodal curves of the different ternary systems formed by AGB-ILs and potassium phosphate buffer at $\mathrm{pH}$ 7.0, in an orthogonal representation. The experimental weight fraction data are given in the ESI. + The values of the fitting parameters and equation, as well the experimental TLs, TLLs, and volume ratio (VR), are provided in the ESIt.

All ILs investigated are able to form ABS with the phosphate buffer salt at $\mathrm{pH} 7.0$, with all compositions above the respective binodal curve resulting in ABS or TPP systems that can be used in separation processes, as in the current work for the purification and recovery of mAbs. The amount required to reach 100 wt\% corresponds to the content of water or supernatant that needs to be added to each system.

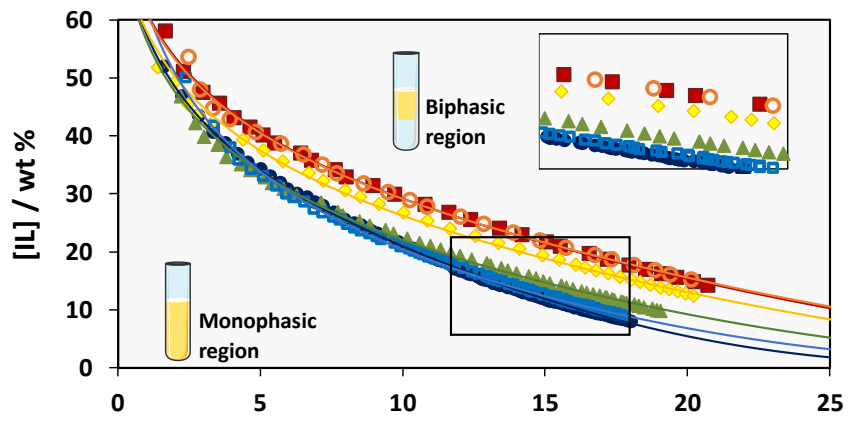

$\left[\mathrm{KH}_{2} \mathrm{PO}_{4} / \mathrm{K}_{2} \mathrm{HPO}_{4} \mathrm{pH} 7\right] /$ wt \%

Figure 2. Phase diagrams of the $\mathrm{ABS}$ composed of $\mathrm{IL}+$ $\mathrm{KH}_{2} \mathrm{PO}_{4} / \mathrm{K}_{2} \mathrm{HPO}_{4}+\mathrm{H}_{2} \mathrm{O}$ at $\mathrm{pH} 7.0$ in weight fraction percentage: $\left[\mathrm{Et}_{3} \mathrm{NC}_{4}\right] \mathrm{Br}(\diamond) ;\left[\mathrm{Bu}_{3} \mathrm{NC}_{4}\right] \mathrm{Br}(\bullet) ;\left[\mathrm{Pr}_{3} \mathrm{NC}_{4}\right] \mathrm{Br}(\Delta)$; $\left[\mathrm{MepyrNC} \mathrm{C}_{4}\right] \mathrm{Br}$ (吕); $\left[\mathrm{N}_{4444}\right] \mathrm{Br}(\square) ;\left[\mathrm{C}_{4} \mathrm{mim}\right] \mathrm{Br}(\bigcirc)$.

A larger biphasic region indicates a higher ability of the IL to form two immiscible phases (biphasic system); e.g. at $15 \mathrm{wt} \%$ of salt, the following trend for $A B S$ formation was found: $\left[\mathrm{C}_{4} \mathrm{mim}\right] \mathrm{Br} \approx\left[\mathrm{MepyrNC}{ }_{4}\right] \mathrm{Br}<\left[\mathrm{Et}_{3} \mathrm{NC}_{4}\right] \mathrm{Br}<\left[\mathrm{Pr}_{3} \mathrm{NC}_{4}\right] \mathrm{Br}<\left[\mathrm{N}_{4444}\right] \mathrm{Br}$ $<\left[\mathrm{Bu}_{3} \mathrm{NC}_{4}\right] \mathrm{Br}$. All ILs share the same anion, being this trend a direct result of the IL cation chemical structure and its affinity for water. IL cations with lower affinity for water, i.e. with longer aliphatic chains or with no aromatic groups, form ABS more easily. The trend obtained indicates the preferential formation of hydration complexes of the salt ions, leading to the IL salting out, and in agreement with literature. ${ }^{18}$ Furthermore, compared to the widely investigated IL $\left[\mathrm{C}_{4} \mathrm{mim}\right] \mathrm{Br}$, it is here shown that AGB-ILs require lower amounts of IL and/or salt to create ABS and consequently TPP systems, which is beneficial when envisioning the development of low-cost and sustainable separation processes. It should be remarked that these AGB-ILs have been previously described as harmless or practically harmless toward the marine bacteria Allvibrio fischeri. ${ }^{34}$ Even when compared to a more hydrophobic and commercial IL, such as $\left[\mathrm{N}_{4444}\right] \mathrm{Br}$, the $\mathrm{AGB}-\mathrm{IL}\left[\mathrm{Bu}_{3} \mathrm{NC}_{4}\right] \mathrm{Br}$ performs better, requiring lower amounts to undergo liquid-liquid demixing.
Purification and recovery of anti-IL-8 mAbs by ABS and TPP strategies

The qualitative and quantitative characterization of the $\mathrm{CHO}$ cell culture supernatants was initially performed to appraise the complexity of the medium from which anti-IL-8 mAbs aimed to be purified. The SDS-PAGE under reducing conditions and SEHPLC characterization results of the $\mathrm{CHO}$ cell culture supernatant are provided in Figure 3.

A
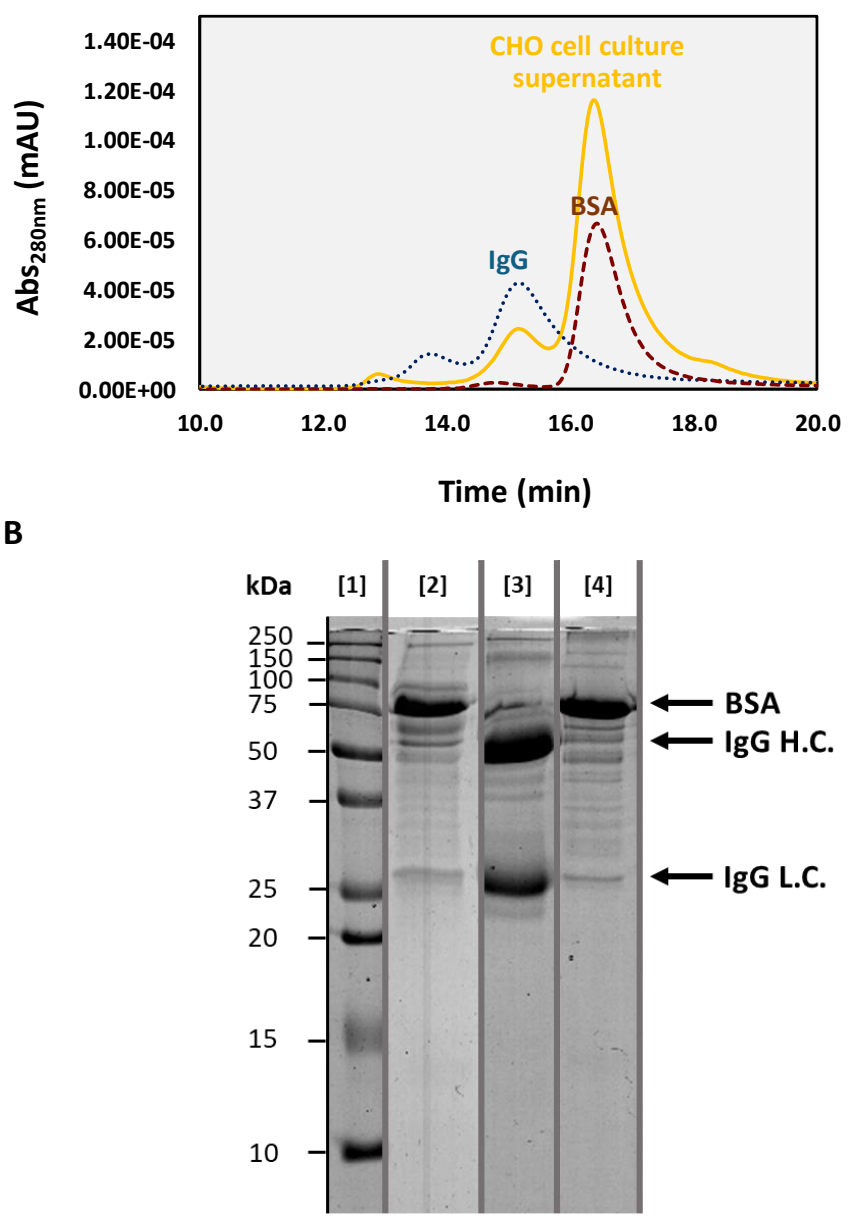

Figure 3. Characterization of the $\mathrm{CHO}$ cell culture supernatant: (A) SE-HPLC chromatograms of the $\mathrm{CHO}$ cell culture supernatant $(-)$, pure IgG solution $100 \mathrm{mg} \cdot \mathrm{L}^{-1}(\cdot \cdot)$, and pure BSA solution 200 $\mathrm{mg} \cdot \mathrm{L}^{-1}(--)$; (B) SDS-PAGE gel: lane 1 - molecular weight marker; lane $2-\mathrm{CHO}$ cell culture supernatant; lane 3 - pure IgG solution $1 \mathrm{~g} \cdot \mathrm{L}^{-1}$; lane 4 - pure BSA solution $1 \mathrm{~g} \cdot \mathrm{L}^{-1}$. BSA (red) and IgG (green) heavy (H.C.) and light (L.C.) chains are identified.

Under the chromatographic conditions used, pure IgG samples present 2 chromatographic peaks: one corresponding to the IgG monomer and the other to IgG aggregates, with a retention time of $c a .15 .0 \mathrm{~min}$ and $13.8 \mathrm{~min}$, respectively. IgG is composed of 2 heavy chains with a molecular weight of $50 \mathrm{kDa}$, and 2 light chains with a molecular weight of $25 \mathrm{kDa}$, visible in the reduced SDS-PAGE results (Figure 3 (b)). The major protein impurity in the feed is bovine serum albumin (BSA), with a retention time 
of ca. $16.8 \mathrm{~min}$ and a molecular weight of $66.5 \mathrm{kDa}$. Apart from IgG and BSA, CHO cell culture supernatants also present other protein impurities resulting from the medium used in the cell proliferation and growth, and also from their metabolism (e.g. insulin, transferrin and other $\mathrm{CHO}$ host cell proteins), which may correspond to the lighter protein corresponding bands in Figure 3.

The cell culture was maintained for several months, resulting in a natural variation of the proteins content between each feedstock. The anti-IL-8 mAbs concentration varied between 40.5 and $99.4 \mathrm{mg} \cdot \mathrm{L}^{-1}$, with an average content of $67.7 \pm 23.7$ $\mathrm{mg} \cdot \mathrm{L}^{-1}$ and an average purity of $16.9 \pm 3.8 \%$. However, in all cases where ABS and TPP systems were applied, the original feedstock was always analyzed to infer the improvements achieved in respect to the original feedstock.

After the characterization of the phase diagrams and of the mAbs-containing feedstock, the potential of IL-based ABS as purification routes for anti-IL-8 directly from $\mathrm{CHO}$ cell culture supernatants was addressed for three mixture compositions (15 wt $\% \mathrm{KH}_{2} \mathrm{PO}_{4} / \mathrm{K}_{2} \mathrm{HPO}_{4} \mathrm{pH} 7.0+22.5 \mathrm{wt} \% \mathrm{H}_{2} \mathrm{O}+37.5 \mathrm{wt} \% \mathrm{CHO}$ cell culture supernatant + IL ranging from $25 \mathrm{wt} \%$ to $40 \mathrm{wt} \%)$. The performance of all ABS was investigated in terms of recovery yield (\% Yield $\left.d_{l g G}\right)$ and purification factor $\left(P F_{l g G}\right)$, whose results are shown in Figure 4 . The use of the purification factor allows to reduce discrepancies in the results derived from the variability of the biological original matrix, and is valuable to carry out a first screening of the several AGB-IL-based systems performance. The composition of each phase for the mixture points used in separation processes, detailed data of the recovery yields and purification factors are given in the ESIt.

For the mixture composition with a lower IL content ( $25 \mathrm{wt} \%$ ), a preferential partition of all proteins to the top (IL-rich) phase was observed, for all ILs (Figure 4 (A)). This phenomenon may be explained by the salting-out effect induced by the phosphate-based salt, promoting the exclusion of proteins into the opposite phase. Nevertheless, biomolecules affinity and interactions with ILs play a significant role since this behavior does not commonly occur in polymer-salt $A B S^{38}$, where the salt also acts as a salting-out agent. In IL-based ABS, a multitude of specific interactions (van der Waals, hydrogen-bonding and electrostatic interactions) occurring between the amino acids residues at the proteins surface and ILs seem to govern their partitioning to the IL-rich phase, as previously reported with other proteins. ${ }^{39-41}$ The isoelectric point (pl) of anti-IL-8 is $c a$. $9.3,35$ and as the $\mathrm{pH}$ of the investigated IL-based systems is 7.0, antibodies are positively charged and electrostatic interactions may play a role. Nevertheless, if they were the most relevant type of interactions, IgG should preferentially partition to the salt-rich phase, as typically observed in polymer-salt ABS. ${ }^{38,43}$ In this work, the partition of IgG is triggered to the top phase of ABS using ILs instead of polymers, which may be due to preferential hydrogen-bonding and dispersive forces between the target protein and ILs.

When using 25 wt\% of IL in the ABS composition, recovery yields of mAbs ranging from $73.1 \%$ up to $100.0 \%$ (for [ $\left.\mathrm{Bu}_{3} \mathrm{NC}_{4}\right] \mathrm{Br}$ ) are obtained in a single step, with purification factors ranging from 1.0 to 1.5. Moreover, all AGB-IL-based ABS composed of $25 \mathrm{wt} \%$ of IL allow a high recovery of mAbs (>92\%), performing better than the commercial ILs that lead to a maximum recovery yield of $73.1 \%$. mAbs losses with these ILs are probably associated to precipitation since some turbidity was detected in these systems. Overall, the best results were obtained using the ABS composed of $\left[\mathrm{Bu}_{3} \mathrm{NC}_{4}\right] \mathrm{Br}$, allowing the complete recovery of the antibodies in a single-step, with a purification factor of $1.5 \pm 0.1$. According to the recovery yields achieved, it is clear that the IL cation core and the respective alkyl side chain length have a significant influence on the mAbs partitioning between the phases and in the maintenance of their structure.

A

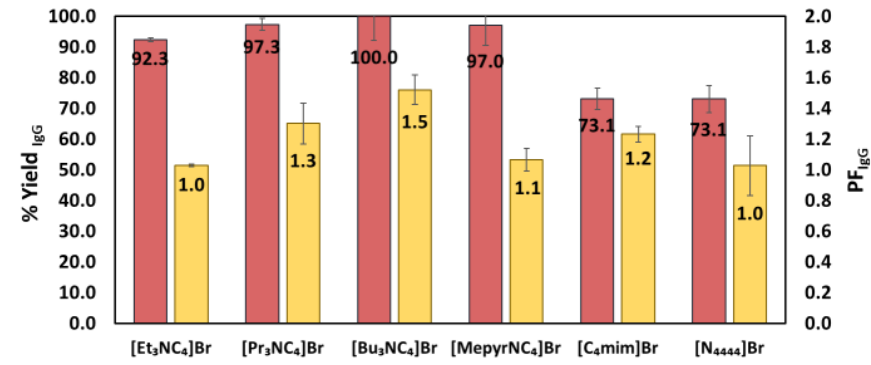

B

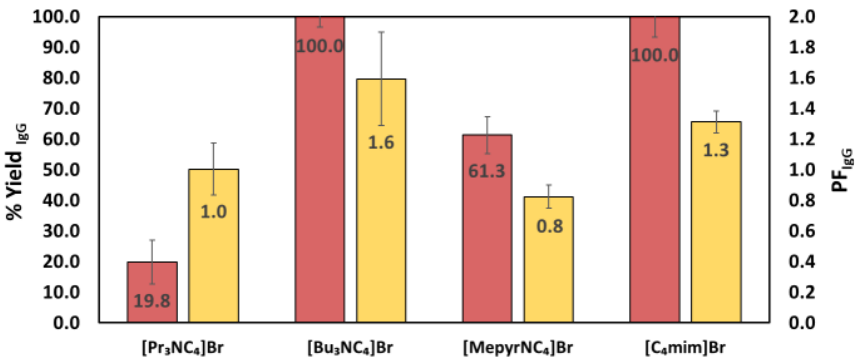

C

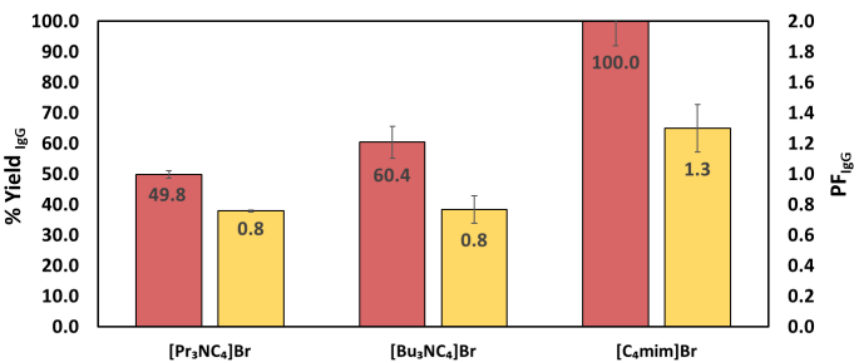

Figure 4. Recovery yields (\%Yield ${ }_{l g G}-\square$ ) and purification factors $\left(P F_{l g G}-\square\right)$ of anti-IL-8 mAbs in the IL-rich phase using ABS composed of IL +15 wt\% $\mathrm{KH}_{2} \mathrm{PO}_{4} / \mathrm{K}_{2} \mathrm{HPO}_{4} \mathrm{pH} 7+22.5$ wt\% $\mathrm{H}_{2} \mathrm{O}$ + 37.5 wt $\%$ CHO cell culture supernatant: (A) 25 wt\% IL; (B) 30 $w t \%$ IL; (C) 40 wt\% IL.

The separation of biomolecules from a complex mixture in ABS can be manipulated by various factors, e.g. by the nature of the phase-forming components and their concentrations. This last possibility was taken into account, conducted by increasing the concentration of the IL from $25 \mathrm{wt} \%$ to $30 \mathrm{wt} \%$ and $40 \mathrm{wt} \%$ while maintaining the composition of the salt at $15 \mathrm{wt} \%$. The goal is to optimize the purification process with four ILs that led to the most promising results in the first screening $\left(\left[\mathrm{Pr}_{3} \mathrm{NC}_{4}\right] \mathrm{Br}\right.$, 
$\left[\mathrm{Bu}_{3} \mathrm{NC}_{4}\right] \mathrm{Br}$, [MepyrNC $\left.\mathrm{M}_{4}\right] \mathrm{Br}$ and $\left.\left[\mathrm{C}_{4} \mathrm{mim}\right] \mathrm{Br}\right)$. The results obtained are depicted in Figure $4((\mathrm{~B})$ and $(\mathrm{C}))$.

The recovery yields for IgG of the systems composed of $30 \mathrm{wt} \%$ of IL range from $19.8 \%$ to $100 \%$; however, when $40 \mathrm{wt} \%$ of IL is used (Figure $4(C)$ ), recovery yields ranging from $49.8 \%$ to $100 \%$ are obtained at the IL-rich phase. In general, ABS formed by $\left[\mathrm{Pr}_{3} \mathrm{NC}_{4}\right] \mathrm{Br}$ present the lowest performance regarding the IgG recovery in the top phase. Similarly, ABS composed of $30 \mathrm{wt} \%$ of [ $\left.\mathrm{MepyrNC}_{4}\right] \mathrm{Br}$ lead to a decrease in the anti-IL-8 recovery and purification factor $(61.3 \pm 6.1 \%$ and $0.8 \pm 0.1$, respectively), being these two ILs discarded for the evaluation with $40 \mathrm{wt} \%$ of IL. For comparison purposes, the systems composed of the commercial IL [ $\left.\mathrm{C}_{4} \mathrm{mim}\right] \mathrm{Br}$ were studied. These systems allow the complete recovery of anti-IL- $8 \mathrm{mAbs}$ with the increase of the IL concentration up to $40 \mathrm{wt} \%$, with a purification factor of 1.30 in both cases. Regarding AGB-ILs, ABS composed of $\left[\mathrm{Pr}_{3} \mathrm{NC}_{4}\right] \mathrm{Br}$ and [MepyrNC $\left.\mathrm{N}_{4}\right] \mathrm{Br}$ are not suitable to purify the target biomolecule from the complex medium under any concentration of IL (from $25 \mathrm{wt} \%$ to $40 \mathrm{wt} \%$ ). The most promising ABS is constituted by $\left[\mathrm{Bu}_{3} \mathrm{NC}_{4}\right] \mathrm{Br}$ at $30 \mathrm{wt} \%$, where recovery yields of $100 \%$ and purification factors of 1.6 are achieved in one-step. Overall, the most hydrophilic ILs, which present smaller biphasic regions in their phase diagrams and shorter TLs, are the ones that lead to the less promising results (e.g. $\left[\mathrm{Pr}_{3} \mathrm{NC}_{4}\right] \mathrm{Br}$ and $\left[\mathrm{MepyrNC} \mathrm{C}_{4}\right] \mathrm{Br}$ ). On the other hand, more hydrophobic ILs (e.g. $\left[\mathrm{Bu}_{3} \mathrm{NC}_{4}\right] \mathrm{Br}$, but still miscible in water), with larger biphasic regions and TLs, are more efficient options to recover and purify mAbs.

In addition to the IgG recovered at the IL-rich phase, the best identified $\mathrm{ABS}$ composed of $\left[\mathrm{Bu}_{3} \mathrm{NC}_{4}\right] \mathrm{Br}$ also leads to the creation of a protein-rich interface precipitate ( $c f$. the ESI+), particularly visible at the higher IL concentrations (40 wt\%) and responsible for the lower recovery yields shown in Figure 4 (C). This phenomenon is related with the water content in the top phase, which decreases as the IL concentration increases (cf. the $\mathrm{ESI}^{+}$), thus not allowing the complete solubilization of proteins and resulting in the formation of ILTPP systems. Mixture compositions comprising higher concentrations of IL, corresponding to longer tie-lines, imply higher concentrations of salt in the bottom phase. Due to the salt ions preferential solvation, less water will be available for the proteins solvation, resulting in the exposure of hydrophobic patches which promotes protein-protein interactions that ultimately lead to the proteins precipitation. This salting-out phenomenon has been reported for the precipitation of food proteins at the interface of IL-salt ABS, defined as ILTPP, although no complex and real mixtures have been addressed. ${ }^{25,} 26$

Curiously, antibodies precipitation in ABS has been systematically considered as a "bad feature" in the literature and, consequently, ABS promoting IgG precipitation are usually discarded due to their "low performance". ${ }^{43,46-50}$ Nevertheless, if precipitation is selective and does not compromise the antibodies stability and activity, this approach should be considered as an alternative and effective IgG downstream strategy. Accordingly, the presence of a significant amount of precipitate in $A B S$ composed of higher amounts (40 wt\%) of $\left[\mathrm{Bu}_{3} \mathrm{NC}_{4}\right] \mathrm{Br}$ encouraged the investigation of the ILTPP approach as a potential strategy to purify and recover mAbs.
The proteins precipitate formed between the two coexisting phases in the ABS was recovered, resuspended in an aqueous solution of PBS ( $\left.\mathrm{pH} 7.0,100 \mathrm{mmol} \cdot \mathrm{L}^{-1}\right)$ and further analyzed. Through this approach and at the conditions under discussion, there is the selective (one-step) precipitation of IgG directly from the $\mathrm{CHO}$ cell culture supernatant, with a recovery yield of $41.0 \pm 2.6 \%$ and a purification factor of $2.7 \pm 0.1$ (\% Purity $_{\lg G}=$ $60.9 \pm 2.0 \%)$. Overall, with the ABS formed by 40 wt \% of $\left[\mathrm{Bu}_{3} \mathrm{NC}_{4}\right] \mathrm{Br}, 60.4 \pm 5.2 \%$ of $\operatorname{lgG}$ with a purification factor of $0.8 \pm$ 0.1 is recovered in the IL-rich phase, whereas the remaining antibody is recovered at the ABS interface with a recovery yield of $41.0 \pm 2.6 \%$ and a purification factor of $2.7 \pm 0.1$. In Figure 5 , the SDS-PAGE gel of all the recovered fractions is provided, allowing not only to infer the mAbs integrity, but also to corroborate the discussed purification results. It should be remarked that although promising results were obtained with higher concentrations of IL, higher concentrations of IL (> 40 wt\%) are not experimentally feasible given the amount of feedstock that needs to be loaded to create ABS.

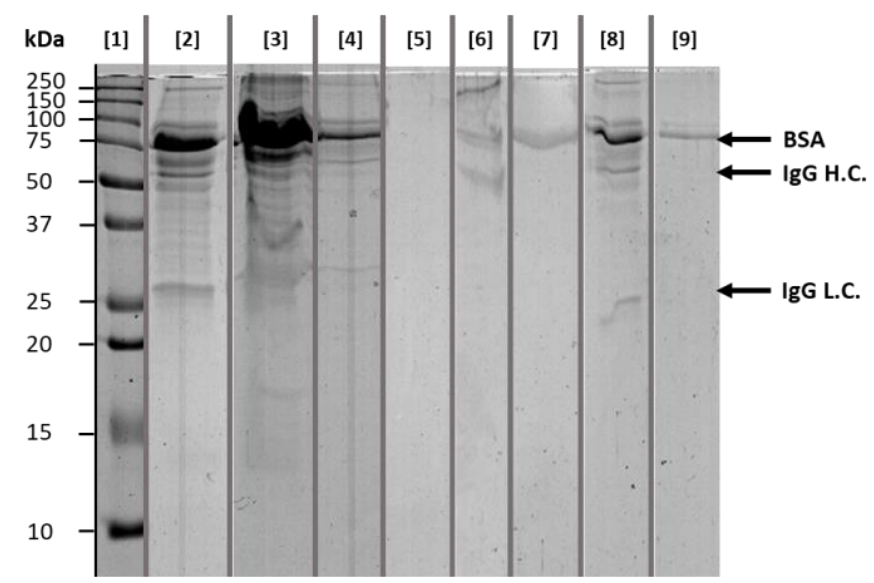

Figure 5. SDS-PAGE of the recovered fractions using the system composed of 40 wt\% $\left[\mathrm{Bu}_{3} \mathrm{NC}_{4}\right] \mathrm{Br}+15 \mathrm{wt} \% \mathrm{KH}_{2} \mathrm{PO}_{4} / \mathrm{K}_{2} \mathrm{HPO}_{4} \mathrm{pH}$ $7.0+22.5$ wt $\% \mathrm{H}_{2} \mathrm{O}+37.5$ wt\% $\mathrm{CHO}$ cell culture supernatant (ABS fractions + UF fractions). Lane 1 -molecular weight marker (kDa); lane $2-\mathrm{CHO}$ cell culture supernatant; lane 3 - ABS top phase; lane 4 - ABS precipitate; lane 5 - ABS bottom phase; lane 6 - UF retentate of top phase; lane 7 - UF filtrate of top phase; lane 8 - UF retentate of precipitate; lane 9 - UF filtrate of precipitate. The bands corresponding to BSA, IgG heavy chain (H.C.) and IgG light chain (L.C.) are also labeled.

To fully characterize the potential of ABS and TPP as IgG onestep purification platforms, we evaluated the biological activity of the anti-IL- $8 \mathrm{mAbs}$ in the IL-rich phase and in the precipitate by competitive ELISA, which was found to be $90.5 \pm 2.0 \%$ and $74.7 \pm 3.2$, respectively. The detailed mAbs activity results are given in the ESIt. The mAbs activity in the IL-rich phase is similar to that presented by the antibodies in the $\mathrm{CHO}$ cell culture supernatant before the purification process (90.6 $\pm 1.3 \%)$, meaning that the extraction using the IL-based approach does not affect or decrease the anti-IL-8 biological activity. Despite the remarkable results obtained with ILTPP, the anti-IL-8 activity slightly decreases in the precipitate fraction, probably due to 
structural changes induced by precipitation, but still in the order of that reported for anti-IL-8 purification using ProA affinity chromatography $79 \pm 9 \%){ }^{35}$ Since the biological activity of biomolecules is closely related with their 3D structure, these observations allowed us to infer that minimal conformational changes occur with IL-based ABS and TPP systems.

\section{Hybrid processes and IgG recovery}

Aiming the IgG recovery from the IL-rich phase and the IL removal from the antibodies' fractions, ultrafiltration (UF) was finally applied, allowing us to propose the use of hybrid processes to purify IgG by combining ABS + UF and TPP + UF purification, recovery and final formulation of mAbs in phosphate-based solutions can be performed, is given in Figure 6.

The UF step of the top IL-rich phase allows to recover $47.3 \pm$ $13.5 \%$ of IgG in the retentate, with an increase of $88.5 \%$ in the purity level, up to $32.8 \pm 1.5 \%$ vs. $17.4 \pm 2.0 \%$ before UF, and with an overall IgG recovery yield of $28.6 \pm 18.7 \%$ in route (i). The IgG recovery yield in the filtrate is $45.1 \pm 16.1 \%$, presenting an IgG purity of $29.8 \pm 5.7 \%$, and with an overall yield of the twostep process (route (ii)) of $27.2 \pm 21.3 \%$, with the anti-IL- 8 mAbs biological activity of $66.4 \pm 2.4 \%$. Nevertheless, it should be remarked that the filtrate is the fraction where the $\mathrm{IL}$ is also

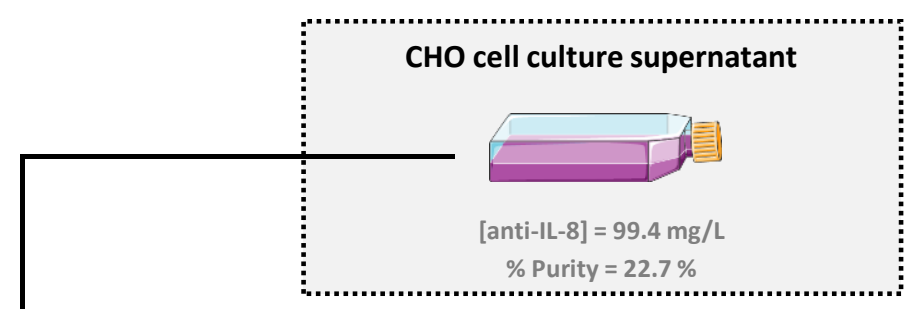

AQUEOUS BIPHASIC SYSTEM/ THREE-PHASE PARTITIONING
ULTRAFILTRATION
FINAL PRODUCT

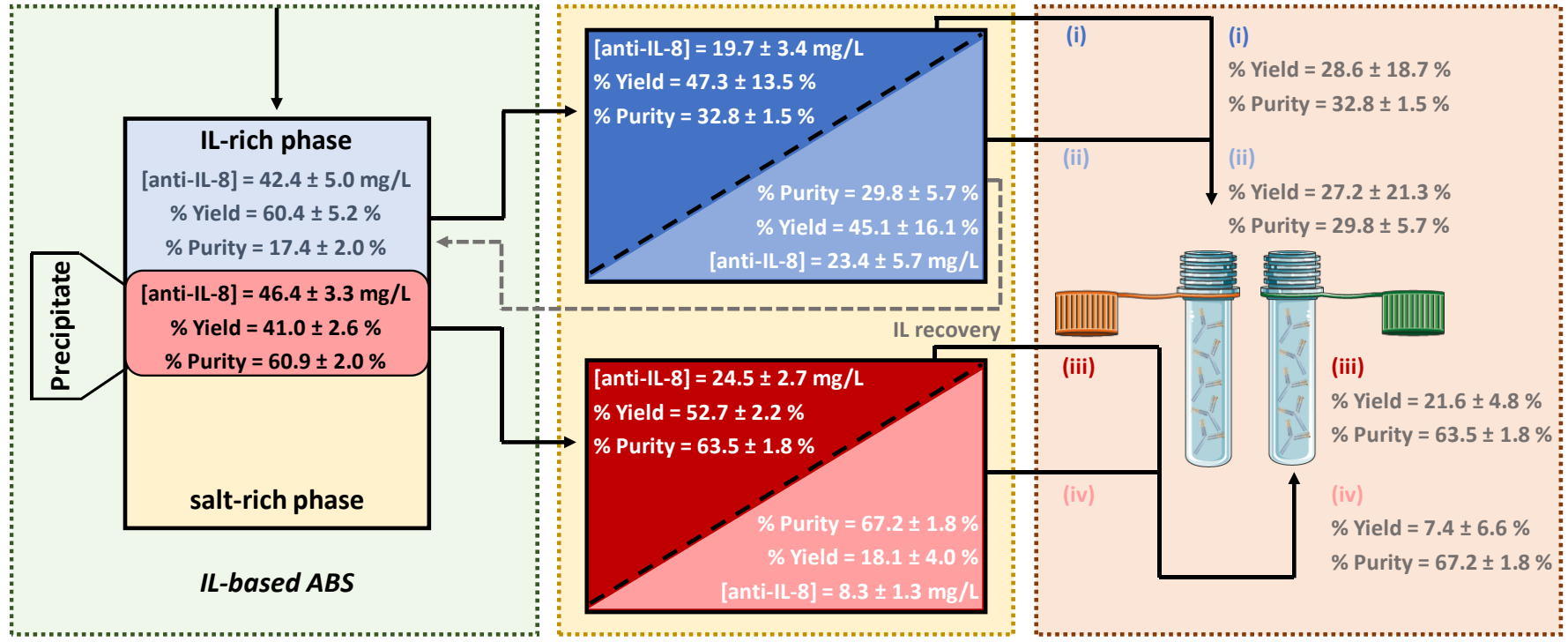

Figure 6. Schematic representation of the integrated and hybrid processes developed for mAbs downstream processing; routes to recover IgG with different purity levels and allowing the IL recover and reuse are identified. steps. UF also allows to recover antibodies in a biological buffer, viable for the envisioned IgG therapeutic applications, and to recover the IL for further use. As a proof of concept, an ultrafiltration step was applied to the IL-rich phase and precipitate containing IgG of the most promising identified $A B S$ (composed of $40 w t \%\left[\mathrm{Bu}_{3} \mathrm{NC}_{4}\right] \mathrm{Br}+15 \mathrm{wt} \% \mathrm{KH}_{2} \mathrm{PO}_{4} / \mathrm{K}_{2} \mathrm{HPO}_{4} \mathrm{pH}$ $7.0+7.5$ wt $\% \mathrm{H}_{2} \mathrm{O}+37.5$ wt $\% \mathrm{CHO}$ cell culture supernatant). The performance of this additional step was investigated in terms of IgG concentration ([IgG]), recovery yield (\% Yield $\left.{ }_{\mid g G}\right)$, purity level (\% Purity ${ }_{I g G}$ ) and purification factor (PF). These parameters were determined for the different strategies that could be combined: (i) TOP phase + UF retentate; (ii) TOP phase + UF filtrate; (iii) Precipitate + UF retentate; (iv) Precipitate + UF filtrate. Detailed data are given in the ESIt. A summary of the results, while highlighting the developed integrated processes in which the present, and therefore the choice of using this fraction for IgG formulation or for IL recovery for further reuse should be made based on the final desired application.

Despite the good results accomplished using the ABS IL-rich phase, an even better performance was achieved with the ILTPP approach combined with ultrafiltration. The IgG recovery yield in the retentate is $52.7 \pm 2.2 \%$, with a purity level of $63.5 \pm 1.8 \%$, whereas the overall recovery yield of the two steps is $21.6 \pm$ $4.8 \%$ (overall process/route (iii)). The UF step in the filtrate results in a low IgG recovery yield of $18.1 \pm 4.0 \%$ (that only results in an overall yield of the two-step process of $7.4 \pm 6.6 \%$ ); however, this approach leads to an increase in the purity level up to $67.2 \pm 1.8 \%$ (route (iv)).

The proteins profile of each UF fraction is provided in Figure 5, in which is possible to identify that, as expected, IgG is mainly 
retained in the retentate fractions. It is important to remark that IgG is present in all fractions evaluated, as shown in the SEHPLC chromatograms (in the ESIt), even though in lower concentrations. After UF the peak corresponding to low molecular weight protein impurities is only present in the filtrate, meaning that with this step we completely remove one class of protein impurities, representing the basis for the increment of $88.5 \%$ in the purity level of IgG in the retentate fraction (comparing to the top IL-rich phase fraction). The precipitate fraction after UF does not contain protein impurities with lower molecular weights (that are only retained in the top phase of the system), supporting the high purity level attained with this fraction (up to $67.2 \%$ ). Although a membrane with a $100 \mathrm{kDa}$ cut-off was used, some 3D conformation of the antibodies allow, in some extension, their passage through the membrane, thus leading to some losses in the IgG recovery yield. This step is however of high relevance since the IL can be recovered and reused, and IgG can be recovered in a phosphate aqueous solutions viable to proceed to biotechnological and health related applications.

Based on the overall results obtained, an hybrid and integrated purification platform is proposed to purify mAbs from $\mathrm{CHO}$ cell culture supernatants, comprising the following steps: (1) ILbased ABS and TPP for mAbs recovery and purification; (2) UF for mAbs polishing and IL removal/salt exchange; and (3) a final product formulation through two routes, according to the IgG desired purity. Based on the combination of the fractions with similar purity levels summarized in Figure 6 , such as routes (i) + (ii) and (iii) + (iv), two fractions of anti-IL-8 mAbs can be recovered with purity levels higher than $29 \%$ and $63 \%$, respectively. Although further optimization studies can be carried out aiming at achieving the complete precipitation of mAbs at the ABS interface, as well as the testing of other membrane cut-offs, the developed platforms reveal a high potential and provide new directions in the design of more sustainable IL-based strategies for the purification and recovery of biopharmaceuticals such as mAbs.

\section{Conclusions}

The use of IL-based purification and recovery strategies for mAbs directly from $\mathrm{CHO}$ cell culture supernatants was here proposed and evaluated for the first time. To this end, novel ABS composed of glycine-betaine analogues ILs and $\mathrm{K}_{2} \mathrm{HPO}_{4} / \mathrm{KH}_{2} \mathrm{PO}_{4}$ at $\mathrm{pH}$ 7.0, the respective three-phase partitioning systems, and hybrid processes combined with ultrafiltration, were investigated. With the studied $A B S, m A b s$ preferentially partition to the IL-rich phase, with recovery yields up to $100 \%$ and purification factors up to 1.6. With IL-based three-phase partitioning approaches a precipitate enriched in mAbs is obtained at the ABS interface, yielding $41.0 \%$ of $\operatorname{lgG}$ with a purification factor of 2.7 (purity of $60.9 \%$ achieved in a singlestep). The biological activity of anti-IL- 8 mAbs is maintained after the several purification and recovery steps, being superior and competitive with the activity of mAbs purified by ProA chromatography. Hybrid processes combining the two previous techniques and an ultrafiltration step were finally applied and evaluated, allowing the recovery of mAbs from the different fractions in an appropriate buffer solution for biopharmaceuticals formulation, while allowing the simultaneous IL removal and reuse. The best results were obtained with the hybrid process combining IL-TPP and ultrafiltration, where IgG with a purity of $67.2 \%$ is obtained.

\section{Conflicts of interest}

There are no conflicts to declare.

\section{Acknowledgements}

This work was developed within the scope of the project CICECO - Aveiro Institute of Materials (UID/CTM/50011/2019), financed by national funds through the FCT/MCTES, and project IL2BioPro (POCI-01-0145-FEDER-030840). The work was also financed by iBB through research unit (UID/BIO/04565/2013) and project PureMab (PTDC/QEQ-PRS/0286/2014). The authors acknowledge the financial support from the European Union Framework Programme for Research and Innovation HORIZON 2020, under the TEAMING Grant agreement No 739572 - The Discoveries CTR. E.V. Capela, A.F.C.S. Rufino and A.P.M. Tavares acknowledge FCT for the PhD grants SFRH/BD/126202/2016 and SFRH/BD/138997/2018, and the research contract IF/01634/2015, respectively. M.M. Pereira acknowledges the Short Term Scientific Mission grant (COST-STSM-ECOST-STSMCM1206-011015-066583) and financial support from COST-IL.. M.G. Freire acknowledges the European Research Council (ERC) for the Starting Grant ERC-2013-StG-337753.

\section{References}

1. E. V. Capela, M. R. Aires-Barros, M. G. Freire and A. M. Azevedo, Frontiers in Clinical Drug Research-Anti Infectives: Volume 4, 2017, 4, 142-203.

2. A. Hey, in Antibodies for Infectious Diseases, American Society of Microbiology, 2015, 3-21.

3. P. Rosa, I. Ferreira, A. Azevedo and M. Aires-Barros, Journal of Chromatography A, 2010, 1217, 2296-2305.

4. D. M. Ecker, S. D. Jones and H. L. Levine, MAbs, 2015, 7, 914.

5. A. L. Grilo and A. Mantalaris, Trends in Biotechnology, 2019, 37, 9-16.

6. M. F. Silva, A. Fernandes-Platzgummer, M. R. Aires-Barros and A. M. Azevedo, Separation and Purification Technology, 2014, 132, 330-335.

7. U. Gottschalk, Biotechnology progress, 2008, 24, 496-503.

8. H. F. Liu, J. Ma, C. Winter and R. Bayer, MAbs, 2010, 2, 480499.

9. A. A. Shukla, B. Hubbard, T. Tressel, S. Guhan and D. Low, Journal of Chromatography B, 2007, 848, 28-39.

10. J. Weinberg, S. Zhang, A. Kirkby, E. Shachar, G. Carta and T. Przybycien, Journal of Chromatography A, 2018, 1546, 8996.

11. S. Sommerfeld and J. Strube, Chemical Engineering and Processing: Process Intensification, 2005, 44, 1123-1137.

12. P.-Å. Albertsson, Nature, 1958, 182, 709. 
13. I. Campos-Pinto, E. V. Capela, A. R. Silva-Santos, M. A Rodríguez, P. R. Gavara, M. Fernandez-Lahore, M. R. AiresBarros and A. M. Azevedo, Journal of Chemical Technology \& Biotechnology, 2018, 93, 1966-1974.

14. I. Campos-Pinto, E. Espitia-Saloma, S. A. Rosa, M. RitoPalomares, O. Aguilar, M. Arévalo-Rodríguez, M. R. AiresBarros and A. M. Azevedo, Separation and Purification Technology, 2017, 173, 129-134.

15. P. Rosa, A. Azevedo, I. Ferreira, S. Sommerfeld, W. Bäcker and M. Aires-Barros, Journal of Chromatography A, 2009, 1216, 8741-8749.

16. A. M. Azevedo, P. A. Rosa, I. F. Ferreira, J. De Vries, T. Visser and M. R. Aires-Barros, Journal of Chromatography B, 2009, 877, 50-58.

17. K. E. Gutowski, G. A. Broker, H. D. Willauer, J. G. Huddleston, R. P. Swatloski, J. D. Holbrey and R. D. Rogers, Journal of the American Chemical Society, 2003, 125, 66326633.

18. M. G. Freire, A. F. M. Claudio, J. M. Araujo, J. A. Coutinho, I. M. Marrucho, J. N. C. Lopes and L. P. N. Rebelo, Chemical Society Reviews, 2012, 41, 4966-4995.

19. A. M. Ferreira, A. F. M. Cláudio, M. Valega, F. Domingues, A. Silvestre, R. D. Rogers, J. A. P. Coutinho and M. G. Freire, Green Chemistry, 2017, 19, 2768 - 2773.

20. E. V. Capela, M. V. Quental, P. Domingues, J. A. Coutinho and M. G. Freire, Green Chemistry, 2017, 19, 1850-1854.

21. A. M. Ferreira, H. Passos, A. Okafuji, A. P. M. Tavares, H. Ohno, M. G Freire and J. A. P. Coutinho, Green Chemistry, 2018, 20, 1218 - 1223.

22. Z. Li, X. Liu, Y. Pei, J. Wang and M. He, Green Chemistry, 2012, 14, 2941-2950.

23. M. V. Quental, M. M. Pereira, A. M. Ferreira, S. N. Pedro, S. Shahriari, A. Mohamadou, J. A. Coutinho and M. G. Freire, Green Chemistry, 2018, 20, 2978-2983.

24. H. Passos, T. B. Dinis, E. V. Capela, M. V. Quental, J. Gomes, J. Resende, P. P. Madeira, M. G. Freire and J. A. Coutinho, Physical Chemistry Chemical Physics, 2018, 20, 8411-8422. E. Alvarez-Guerra and A. Irabien, Separation Science and Technology, 2014, 49, 957-965.

26. E. Alvarez-Guerra and A. Irabien, Journal of Chemical Technology \& Biotechnology, 2015, 90, 939-946.

27. C. Dennison and R. Lovrien, Protein expression and purification, 1997, 11, 149-161.

28

M. Petkovic, K. R. Seddon, L. P. N. Rebelo and C. S. Pereira, Chemical Society Reviews, 2011, 40, 1383-1403.

29. R. A. Sheldon, Green Chemistry, 2017, 19, 18-43.

30. F. De Zwart, S. Slow, R. Payne, M. Lever, P. George, J. Gerrard and S. Chambers, Food Chemistry, 2003, 83, 197204.

S. Huang, L. Mills, B. Mian, C. Tellez, M. McCarty, X.-D. Yang, J. M. Gudas and M. Bar-Eli, The American Journal of Pathology, 2002, 161, 125-134. Teeling, D. Satijn, K. M. Knudsen, E. P. Boot, D. Hudson and O. Baadsgaard, The Journal of Immunology, 2008, 181, 669679.

33. A. Harada, N. Sekido, T. Akahoshi, T. Wada, N. Mukaida and K. Matsushima, Journal of Leukocyte Biology, 1994, 56, 559-564.

34. M. M. Pereira, PhD Thesis, Department of Chemistry, University of Aveiro, 2017.
35. R. dos Santos, S. A. Rosa, M. R. Aires-Barros, A. Tover and A. M. Azevedo, Journal of Chromatography A, 2014, 1355, 115-124.

36. A. M. Ferreira, V. F. Faustino, D. Mondal, J. A. Coutinho and M. G. Freire, Journal of biotechnology, 2016, 236, 166-175.

37. J. C. Merchuk, B. A. Andrews and J. A. Asenjo, Journal of Chromatography B, 1998, 711, 285-293.

38. P. A. Rosa, A. M. Azevedo and M. R. Aires-Barros, Journa of Chromatography A, 2007, 1141, 50-60.

39. M. M. Pereira, S. N. Pedro, M. V. Quental, Á. S. Lima, J. A. Coutinho and M. G. Freire, Journal of Biotechnology, 2015, 206, 17-25.

40. M. Taha, M. V. Quental, I. Correia, M. G. Freire and J. A. Coutinho, Process Biochemistry, 2015, 50, 1158-1166.

A. M. Ferreira, H. Passos, A. Okafuji, A. P. Tavares, H. Ohno, M. G. Freire and J. A. Coutinho, Green Chemistry, 2018, 20, 1218-1223.

42. P. A. Rosa, A. M. Azevedo, S. Sommerfeld, M. Mutter, W. Bäcker and M. R. Aires-Barros, Biotechnology Journal, 2013, 8, 352-362.

43. A. M. Azevedo, P. A. Rosa, I. F. Ferreira and M. R. AiresBarros, Journal of Biotechnology, 2007, 132, 209-217.

44. J. Mündges, J. Zierow and T. Zeiner, Chemical Engineering and Processing: Process Intensification, 2015, 95, 31-42.

45. P. Rosa, A. Azevedo, S. Sommerfeld, W. Bäcker and M. Aires-Barros, Journal of Chromatography B, 2012, 880, 148-156.

46. P. Rosa, A. Azevedo, S. Sommerfeld, M. Mutter, M. AiresBarros and W. Bäcker, Journal of Biotechnology, 2009, 139, 306-313.

47. A. Azevedo, P. Rosa, I. Ferreira and M. Aires-Barros, Journal of Chromatography A, 2008, 1213, 154-161.

48. A. M. Azevedo, A. G. Gomes, P. A. Rosa, I. F. Ferreira, A. M. Pisco and M. R. Aires-Barros, Separation and Purification Technology, 2009, 65, 14-21.

49. B. Andrews, S. Nielsen and J. Asenjo, Bioseparation, 1996, 6, 303-313.

50. F. Hachem, B. Andrews and J. Asenjo, Enzyme and Microbial Technology, 1996, 19, 507-517. 